\title{
Enhanced Magnetocaloric Effect by the Rare Earth Polarization Due to the Exchange with a Transition Metal. Study of $\mathrm{GdCrO}_{4}$
}

Elías PALACIOS ${ }^{1, a}$, , Corrado TOMASI ${ }^{1,2, b}$, Regino SAEZ-PUCHE ${ }^{3, c}$, Antonio J. DOS SANTOS-GARCIA ${ }^{4, d}$, Francisco FERNANDEZ-MARTINEZ ${ }^{4, e}$, and Ramón BURRIEL ${ }^{1, f}$

${ }^{1}$ Instituto de Ciencia de Materiales de Aragón (ICMA) and Dep. de Física de la Materia
Condensada, CSIC-Universidad de Zaragoza, 50009 Zaragoza, Spain

${ }^{2}$ IENI-CNR, Corso Promessi Sposi 29, 23900 Lecco, Italy

${ }^{3}$ Dep. de Química Inorgánica, Universidad Complutense de Madrid, 28040 Madrid Spain

${ }^{4}$ Dep. Ingeniería Mecánica y Química, ETSIDI, Univ. Politécnica de Madrid, 28012 Madrid, Spain

aelias@unizar.es, bcorrado.tomasi@gmail.com, crsp92@quim.ucm.es, dadossant@quim.ucm.es, efrancisco.fernandezm@upm.es, 'burriel@unizar.es

Keywords: Magnetocaloric effect. Mean field. Magnetic refrigeration.

\begin{abstract}
The zircon polymorph of $\mathrm{GdCrO}_{4}$ has a large magnetocaloric effect over a wide temperature range, with $\left|\Delta S_{T}\right|>20 \mathrm{~J} / \mathrm{kg} \cdot \mathrm{K}$ from $6 \mathrm{~K}$ to $34 \mathrm{~K}$, for a magnetic field of $9 \mathrm{~T}$. This unusual behaviour is very interesting on magnetic refrigeration applications, for liquefying $\mathrm{H}_{2}$ or natural gas. The mean-field approach explains that it is due to the weaker Gd-Cr magnetic exchange relative to the $\mathrm{Cr}-\mathrm{Cr}$ one, while the Gd-Gd exchange is negligible. This possibility has not been sufficiently studied and opens an interesting strategy to design more efficient materials for magnetic refrigeration.
\end{abstract}

\section{Introduction}

Adiabatic demagnetization (used since the 1930's [1]) has revealed as an efficient method of liquefying gases like $\mathrm{H}_{2}$ or natural gas, before transport or storage, and indeed some prototypes have been already developed [2,3]. The magnetocaloric material used for this purpose is: a) A magnetically dense material, paramagnetic in the temperature range of interest (e.g. $\mathrm{Gd}_{3} \mathrm{Ga}_{5} \mathrm{O}_{12}$ (GGG), $\mathrm{Dy}_{3}(\mathrm{Ga}, \mathrm{Al})_{5} \mathrm{O}_{12}(\mathrm{DGAG})$ ); or $b$ ) A ferromagnetic material with $T_{C}$ near the working temperature (e.g. $\mathrm{ErCO}_{2}$ ). The main technical parameter to evaluate the cooling capacity of a material is the isothermal entropy change $\Delta S_{T}$ (usually negative) when a given magnetic field $B$ is applied. In case $a$ ) the material has strong cooling capacity only at very low temperatures, below $10 \mathrm{~K}$, whereas in case b) $\left|\Delta S_{T}\right|$ is only high near $T_{C}$. Liquefying gases requires efficient cooling methods over wide temperature ranges, and multi-stage systems are used. Therefore materials with high $\left|\Delta S_{T}\right|$ over a wide temperature range would be very interesting.

Recently, we proposed an idea for designing such materials [4], based on the fact that $\left|\Delta S_{T}\right|$ of a paramagnet for a given field increase $\Delta B$ is enhanced when a previous effective field $B_{i}$ is already present. This is so because, in a paramagnet, when the magnetization is far from saturation (i.e. for low fields or relatively high temperatures, above $10 \mathrm{~K}),\left|\Delta S_{T}\right|$ is proportional to $\Delta\left(B^{2}\right)=\left(B_{i}+B_{f}\right)\left(B_{f}-\right.$ $\left.B_{i}\right)=\left(B_{i}+B_{f}\right) \Delta B$, being $B_{i}, B_{f}$ the initial and final fields. In a compound containing a rare-earth $R$ plus a transition-metal $M$, this case can occur if the $R-R$ exchange interaction is very weak, the $M-M$ exchange is very strong, and the $R-M$ exchange is intermediate, of the order of working temperature. At the working temperature the magnetic moments of the $M$ atoms are fully saturated and the $R-M$ exchange acts as an external field polarizing the $R$ atoms.

Actually, the idea comes from the 1970's [5] and in the 90's it was tested in $R M O_{3}$ perovskites, mainly in $\mathrm{NdFeO}_{3}$ [6], where the experimental heat capacity and magnetization of the $\mathrm{Nd}$ sublattice 
match perfectly with the prediction for a single ion in a constant field, without any external applied field. The Fe sublattice orders antiferromagnetically at $690 \mathrm{~K}$ and the staggered polarizing field on the $\mathrm{Nd}$ sublattice does not produce any net magnetization, but could be observed by neutron diffraction. Therefore, this compound is not useful for magnetic refrigeration. Instead, the zircon polymorphs of $\mathrm{RCrO}_{4}$ compounds are usually ferromagnetic at low temperature. The polarization of the $R$ atom by the exchange should produce a stronger influence on the magnetocaloric effect, as compared to other isostructural $R M \mathrm{O}_{4}$ compounds, when $M$ is a non-magnetic atom. In a previous work, [4] we presented the experimental results for $\mathrm{GdCrO}_{4}$, that orders ferromagnetically at $21.3 \mathrm{~K}$ and has $\left|\Delta S_{T}\right|>20 \mathrm{~J} / \mathrm{kg} \cdot \mathrm{K}$ for $9 \mathrm{~T}$ in the broad range, $6 \mathrm{~K}<T<34 \mathrm{~K}$, when other conventional materials, like $\mathrm{ErCo}_{2}$, exceeds this value only between $34 \mathrm{~K}$ and $46 \mathrm{~K}$, the paramagnet GGG only below $14 \mathrm{~K}$, and DGAG (used in prototypes) never reaches it. In that work we did not treat in detail the analysis of the mean-field model.

\section{Mean Field Model (MF)}

The energy per chemical unit in the MF approximation is

$$
\left.\left.\frac{E}{k_{B}}=-z_{R} J_{R R}\left\langle s_{R}\right\rangle^{2}-z_{M} J_{M M}\left\langle s_{M}\right\rangle^{2}-z_{R M} J_{R M} s_{M}\right\rangle s_{R}\right\rangle-\frac{\mu_{B}}{k_{B}}\left(g_{R}\left\langle s_{R}\right\rangle+g_{M}\left\langle s_{M}\right) B,\right.
$$

where $B$ is the external field, $z_{j}$ the numbers of nearest neighbours, $s_{j}$ the spins and $J_{i}$ the exchange constants between $R$ atoms, $M$ atoms, and between $R$ and $M$ atoms. The interesting case occurs when $J_{R R}<<J_{R M}<J_{M M}$, otherwise, if these exchange constants are comparable, both sublattices order simultaneously as in a conventional ferromagnet. For $\mathrm{GdCrO}_{4}, J_{R R}$ is weak $\left(\mathrm{Gd}\right.$ orders at $T_{N}=$ $4.8 \mathrm{~K}$ ) but antiferromagnetic and a MF model should consider several sublattices for the $R$ atoms. For simplicity, we neglect the small exchange $J_{R R}$. The mean fields at the $R$ and $M$ sublattices are:

$$
\left.B_{M}=B+\frac{k_{B}}{\mu_{B} g_{M}}\left(z_{M} J_{M M} s_{M}\right\rangle+z_{R M} J_{R M} s_{R}\right) ; B_{R}=B+\frac{k_{B}}{\mu_{B} g_{R}} z_{R M} J_{R M}\left\langle s_{R}\right.
$$

The average moments of the sublattices, $\mu_{R}=g_{R} \mu_{B}<s_{R}>$ and $\mu_{M}=g_{M} \mu_{B}<s_{M}>$, are obtained by solving the system of coupled equations:

$$
\frac{\left\langle s_{R}\right\rangle}{s_{R}}=B_{s_{R}}\left(\frac{z_{R M} J_{R M}\left\langle s_{M}\right\rangle}{T}+\frac{\mu_{B} g_{R} s_{R} B}{k_{B} T}\right) ; \frac{\left.s_{M}\right\rangle}{s_{M}}=B_{s_{M}}\left(\frac{\left.z_{R M} J_{R M}\left\langle s_{R}\right\rangle+z_{M} J_{M M} s_{M}\right\rangle}{T}+\frac{\mu_{B} g_{M} s_{M}}{k_{B} T} B\right)
$$

where $s_{R}=7 / 2$ for $\mathrm{Gd}^{3+}, s_{M}=1 / 2$ for $\mathrm{Cr}^{5+}$, and $B_{s}(x)$ is the Brillouin function for spin $s$, defined as

$$
B_{S}(x)=\frac{2 s+1}{2 s} \operatorname{coth}\left[\frac{(2 s+1) x}{2 s}\right]-\frac{1}{2 s} \operatorname{coth}\left[\frac{x}{2 s}\right] \text {. }
$$

The canonical partition function is: $Z=\sum_{m_{R}=-s_{R}}^{s_{R}} \exp \left(\frac{g_{R} \mu_{B} m_{R} B_{R}}{k_{B} T}\right) \times \sum_{m_{M}=-s_{M}}^{s_{M}} \exp \left(\frac{g_{T} \mu_{B} m_{M} B_{M}}{k_{B} T}\right)$.

Finally, the molar thermodynamic functions (in eq. (6) $R=N_{A} k_{B}$ is the ideal gas constant) are obtained in the usual way,

$$
U=N_{A} E ; \quad F=-R T \ln Z ; \quad S(T, B)=(-F+U) / T=R \ln Z+U / T ; \quad C_{B}(T, B)=T\left(\frac{\partial S}{\partial T}\right)_{B} .
$$

For the $\mathrm{GdCrO}_{4}$ zircon phase, $R=\mathrm{Gd}^{3+}, M=\mathrm{Cr}^{5+}, z_{R M}=2, z_{M}=z_{R}=4, g_{R}=g_{M}=2$. The exchange constants $J_{M M}=12.5 \mathrm{~K}, J_{R M}=10 \mathrm{~K}$ give $T_{C}=30 \mathrm{~K}$ and values for $M(T)$ and $\left|\Delta S_{T}\right|$ similar to the experimental results (see below).

Fig. 1, left panel, shows the magnetization of the $R$ and $M$ sublattices for applied fields $B=0$ and $5 \mathrm{~T}$. The $M$ sublattice saturates quickly for $T<T_{C}$, but the moment of the $R$ sublattice increases much more slowly, reaching only $4 \mu_{B}$ (quite below the saturation value) at $T=20 \mathrm{~K}=2 / 3 T_{C}$ for $B$ $=0 \mathrm{~T}$. This leaves room to increase $\mu$ below $T_{C}$ when an external field is applied, involving an entropy decrease. Fig. 1, right panel, shows the entropy increments deduced from the model. For $T$ 
$<<T_{C}$ the $M$ sublattice is saturated and the exchange interaction $R-M$ acts as a constant effective field $B_{\text {ex }}=k_{B} Z_{R M} J_{R M}<s_{M}>/\left(\mu_{B} g_{R}\right)$ on the $R$ sublattice, giving the entropy change represented by the pink line, computed for a constant $B_{\mathrm{ex}}=7 \mathrm{~T}$.
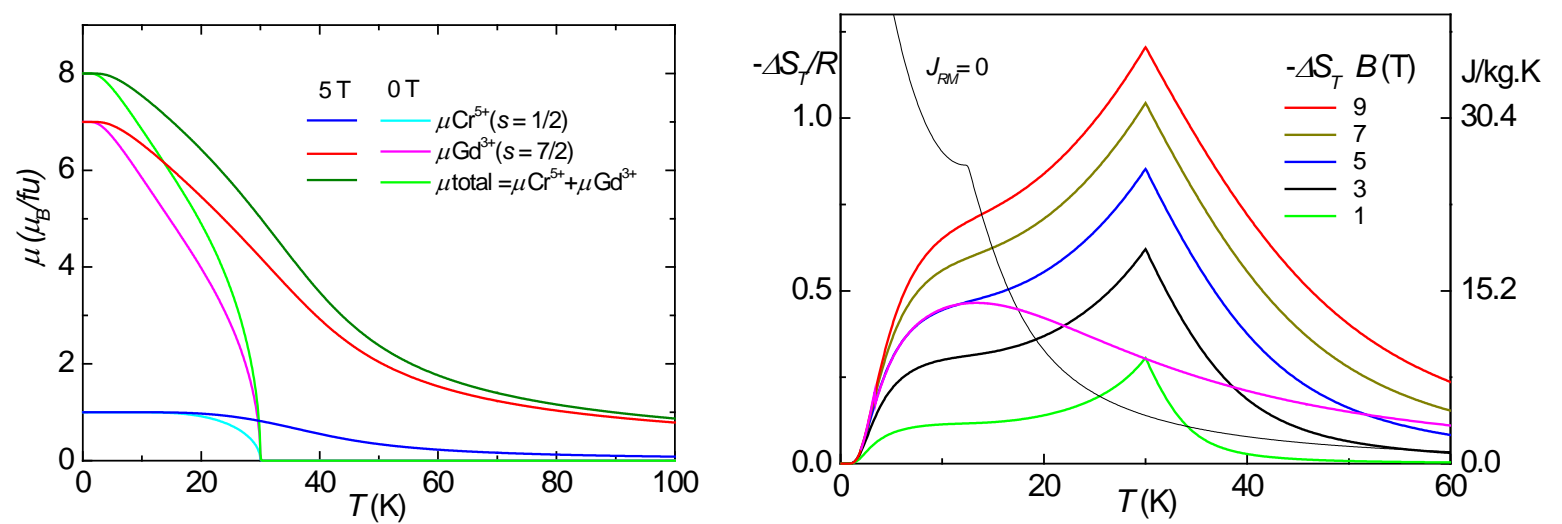

Figure 1. Left: Magnetization of the $R$ and $T$ sublattices, and total for the mean-field model with $J_{M M}=12.5 \mathrm{~K}, J_{R M}=10 \mathrm{~K}$. Right: Isothermal entropy increments $\left|\Delta S_{T}\right|$ from zero to several applied fields, deduced from the model. Pink line: entropy change for a spin $7 / 2$ for $B=5 \mathrm{~T}$ with a constant effective exchange field $B_{\mathrm{ex}}=7 \mathrm{~T}$. Black line: Entropy change for two non-interacting sublattices $R$ and $M$, for $B=5 \mathrm{~T}$ and the same $J_{M M}$ value.

The effect of $B_{\text {ex }}$ produces a shoulder in the curve $\left|\Delta S_{T}\right| v s$. $T$ for a given external field and the blue line in Fig. 1 right collapses with the pink line. For $T \sim T_{C}, B_{\text {ex }}$ is proportional to $<s_{M^{>}}$, which in turn is easily polarized by an external field. Fig. 1, left panel, shows that at $T_{C}=30 \mathrm{~K},\left\langle s_{M}>\right.$ is $82 \%$ of the saturation value for $B=5 \mathrm{~T}$. But the spin of $\mathrm{Gd}^{3+}$ is $7 / 2$ while that of $\mathrm{Cr}^{5+}$ is only $1 / 2$, therefore the entropy increment is higher than expected for $\mathrm{Gd}^{3+}$ in a constant $B_{\text {ex }}$ (pink line) and much higher than for $\mathrm{Cr}^{5+}$ alone, which could only reach $\left|\Delta S_{T}\right| / k_{B}=\ln 2$, for infinite field. The black line shows the calculation for non-interacting $R$ and $M$ sublattices with the same $J_{M M}$ value and $B=$ $5 \mathrm{~T}$. As expected, the $R$ sublattice produces the typical $\left|\Delta S_{T}\right|$ curve for a paramagnet, increasing at very low temperatures. The $M$ sublattice alone would produce the $\left|\Delta S_{T}\right|$ of a usual ferromagnet, with a peak near its corresponding $T_{C}=J_{M M}=12.5 \mathrm{~K}$, but quite low due to the small $\mathrm{Cr}^{5+}$ spin, $1 / 2$, which saturates for fields much lower than $5 \mathrm{~T}$. The $R-M$ interaction increases $T_{C}$ but, more importantly, increases $\left|\Delta S_{T}\right|$. This can be understood since, for instance at $T_{C}$ and zero field the $R$ and $M$ sublattices are unpolarized, but an applied field of $5 \mathrm{~T}$ saturates almost completely the $\mathrm{Cr}^{5+}$ sublattice $\left(\left|\Delta S_{T}\right| \cong k_{B} \ln 2\right)$ and acts on the $\mathrm{Gd}^{3+}$ sublattice as a total effective field $B_{\text {eff }}=B+B_{\text {ex }}=12$ $\mathrm{T}$, giving $\mu_{\mathrm{Gd}}=4.2 \mu_{\mathrm{B}}$. As a result, $\left|\Delta S_{T}\right|$ is high in a wide temperature range. A key detail is that $J_{R M}$ should not be too strong because, in such a case, $\left|\Delta S_{T}\right|$ would be high only near $T_{C}$, but would decay quickly at lower temperatures, since the mean field would saturate the magnetization even without any external field, as happens in a typical ferromagnet.

\section{Experimental}

The experimental data of $\Delta S_{T}$ [4] were obtained in 3 ways: a) From magnetization, via the Maxwell equation, b) From heat capacity at constant field, and c) Directly measured by an original method, based on considerations given in [7]. All the three methods agreed. The small differences between data from magnetization and calorimetry were due to the different demagnetization factors of the samples used. The parameters $J_{R R}$ and $J_{R M}$ were chosen to fit the experimental magnetization, heat capacity and $\Delta S_{T}$ at low temperatures (i.e. $B_{\mathrm{ex}}$ ). The $J_{M M}$ value was chosen from the magnetic energy, given in Eq. 1, compared to the experimental value $\int_{0}^{\infty} C_{m}(T, B=0) d T=258 \mathrm{~J} / \mathrm{mol}$ obtained from the $C_{m}$ data shown in Ref. [4]. With this choice, the experimental $T_{C}$ is lower than the MF 
prediction, as happens in lattices with small number of nearest neighbours. Fig. 2 shows the experimental and MF data of $\Delta S_{T}$ versus the reduced temperature $T / T_{C}$.
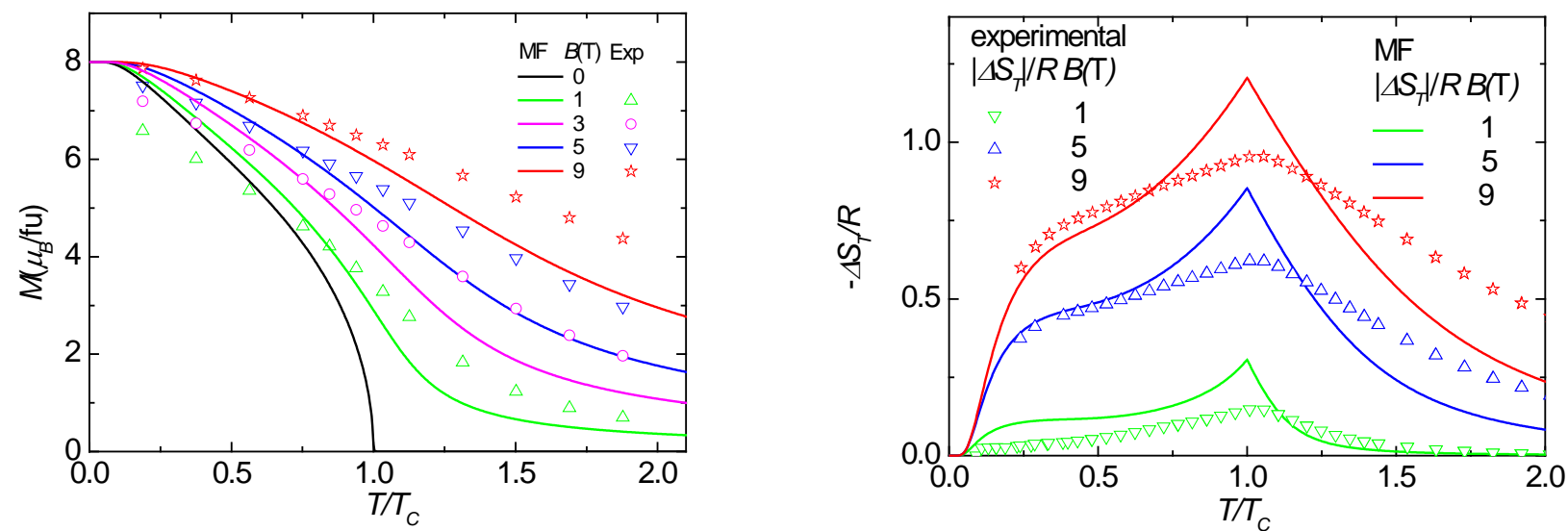

Figure 2: Left: Experimental magnetization of $\mathrm{GdCrO}_{4}$ and $\mathrm{MF}$ calculations vs. reduced temperature. Right: Experimental $\left|\Delta S_{T}\right|$ and calculated curves with the MF model.

The computed data agree quantitatively with the experimental values in the low temperature region, when the $\mathrm{Cr}^{5+}$ moment is saturated. Data for $B=5$ and $9 \mathrm{~T}$ match with the simplification of taking a constant $B_{\text {ex }}=7 \mathrm{~T}$, as occurred in the case of $\mathrm{NdFeO}_{3}$. The values for $B=1 \mathrm{~T}$ do not agree, probably because the demagnetizing field affects significantly the experimental results. At higher temperatures the MF model does not give a quantitative agreement with experimental data, but shows the same general features. There is a shoulder in the low temperature region, a maximum at $T_{C}$, and a slow decrease for $T>T_{C}$. This shoulder produces a wide $T$ range in which $\left|\Delta T_{S}\right|$ is high.

\section{Conclusion}

The mean-field approach gives a qualitative, easily understandable description of the magnetism in zircon $\mathrm{GdCrO}_{4}$, although there is not a detailed quantitative agreement due to the small number of nearest neighbours. The $\mathrm{Cr}^{5+}$ sublattice orders ferromagnetically via exchange interaction at $T_{C}=$ $21.3 \mathrm{~K}$ and polarizes the $\mathrm{Gd}^{3+}$ atoms below this temperature due to a weaker Gd-Cr exchange. This second exchange acts on $\mathrm{Gd}^{3+}$ as an additional effective field, enhancing the magnetocaloric effect at temperatures above $5 \mathrm{~K}$, as compared with isostructural compounds where $\mathrm{Cr}$ is replaced by another non-magnetic atom, like in $\mathrm{GdVO}_{4}$ or $\mathrm{GdAsO}_{4}$. This mechanism provides a high $\left|\Delta S_{T}\right|$ over a wide temperature range, between $6 \mathrm{~K}$ and $34 \mathrm{~K}$, which is of interest regarding to applications in magnetic refrigerators designed for liquefying $\mathrm{H}_{2}$ or natural gas, where the efficiency of other methods decay.

[1] F. Pobell, Matter and Methods at Low Temperatures, $3^{\mathrm{d}}$ ed., Springer, Berlin, 2007.

[2] T. Numazawa, K. Kamiya, T. Utaki, and K. Matsumoto, Cryogenics 62, 185 (2014).

[3] J. Park, S. Jeong, and I. Park, Cryogenics 71, 82 (2015).

[4] E. Palacios, C. Tomasi, R. Sáez-Puche, A. J. Dos santos-García, F. Fernández-Martínez, and R. Burriel, Phys. Rev. B 93, 064420 (2016).

[5] R.M. Hornreich, Y. Komet, R. Nolan, B.M. Wanklyn, and I. Yaeger, Phys. Rev. B 12, 5094 (1975).

[6] J. Bartolomé, E. Palacios, M. D. Kuz’min, and F. Bartolomé, I. Sosnowska, R. Przeniosło, R. Sonntag, and M. M. Lukina, Phys. Rev. B 55, 11432 (1997).

[7 ] G. Lorusso, J.W. Sharples, E. Palacios, O. Roubeau, E.K. Brechin, R. Sessoli, A. Rossin, F. Tuna, E.J.L. McInnes, D. Collison, and M. Evangelisti, Advanced Materials 25, 4653 (2013). 